\title{
No Froissart Bound from Gluon Saturation
}

\author{
Alexander Kovner ${ }^{a}$ and Urs Achim Wiedemann ${ }^{b}$ \\ ${ }^{a}$ Department of Mathematics and Statistics, University of Plymouth, 2 Kirkby Place, Plymouth, PL4 8AA, U.K. \\ ${ }^{b}$ Theory Division, CERN, CH-1211 Geneva 23, Switzerland
}

(Dated: November 3, 2018)

\begin{abstract}
In the previous work [1], we showed that while the nonlinear QCD evolution equation of Balitsky and Kovchegov (BK) leads to saturation of the scattering amplitude locally in impact parameter space, it does not unitarize the total cross section. This result was recently challenged by Ferreiro, Iancu, Itakura and McLerran (FIIM) who claim that the dipole-hadron cross section computed from the BK equation saturates the Froissart bound. In this comment, we point to a fundamental error in the argument of FIIM which invalidates their conclusion. We show that if the total cross section violates unitarity for a coloured scattering probe, it does so also for a colourless scattering probe.
\end{abstract}

In Ref. [1], we demonstrated in two independent ways that the dipole-hadron cross section evolved by the BK equation [3] does not unitarize. The first derivation was given in the target rest frame where the evolution resides in the projectile wave function. This derivation uses explicitly colourless scattering probes. The second derivation was given within a formulation where the evolution is ascribed to the target. This part of the argument can be construed as calculating the scattering probability of a coloured probe. This latter point seems to have caused some confusion. In particular, the authors of Ref. [4] claimed recently that our conclusion holds for coloured probes only and that "for the physically interesting case where the external probe is a (colourless) dipole, there is no problem with unitarity at all." The main purpose of this note is to show that once the Froissart bound is violated for a coloured probe, it is also violated for a colourless scattering probe. We do this by considering the BK evolution directly as the evolution of the target.

In the large $N_{c}$ limit, the BK equation is a closed equation for the $t=\ln 1 / x$ rapidity dependence of the scattering probability $N(x, y)=\frac{1}{N_{c}} \operatorname{Tr}\left\langle 1-U^{\dagger}(x) U(y)\right\rangle$ of a colour singlet dipole with charges at points $x$ and $y$,

$$
\begin{gathered}
\frac{d}{d t} N(x, y)=\bar{\alpha}_{s} \int \frac{d^{2} z}{2 \pi} \frac{(x-y)^{2}}{(x-z)^{2}(y-z)^{2}} \\
{[N(x, z)+N(y, z)-N(x, y)-N(x, z) N(z, y)],}
\end{gathered}
$$

where $\bar{\alpha}_{s}=\frac{\alpha_{s} N_{c}}{\pi}$. The unitary matrix $U(x)$ depends on the colour fields in the target and represents the eikonal factor picked up by the fundamental charge at transverse coordinate $x$ while propagating through the target field.

In the following we concentrate on the evolution of the scattering probability of a dipole of fixed size $r=x-y$ at fixed impact parameter $b=\frac{x+y}{2}$. We will sometimes write $N(r, b)$ instead of $N(x, y)$. Schematically the $x, y$ dependence of $N$ at any given rapidity is depicted on Fig.1. The circle of radius $R_{0}(t)$ bounds what one could call "the interior of the hadron". For impact parameters $b \equiv \frac{x+y}{2}>R_{0}(t)$ the scattering probability is vanishingly small for dipoles of any size. The radius $R\left(t, Q^{2}\right)$ bounds the region inside which $N(r, b) \propto 1$ for fixed $r^{2}=Q^{-2}$ and outside which $N(r, b) \ll 1$. More precise definitions of $R_{0}(t)$ and $R\left(t, Q^{2}\right)$ will be given shortly. The total cross section for the scattering of a dipole of size $Q^{-1}$ on the target is related to $R\left(t, Q^{2}\right)$ as

$$
\sigma\left(t, Q^{2}\right)=2 \int d^{2} b N\left(t, b, Q^{-1}\right) \sim 2 \pi R^{2}\left(t, Q^{2}\right)
$$

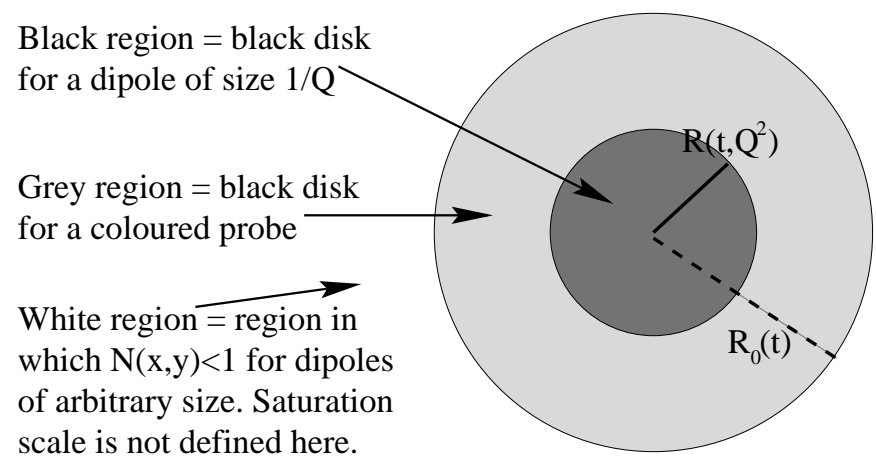

FIG. 1: The three impact parameter regions of a hadronic target, relevant for the total dipole-hadron cross section

We follow the expansion of $R_{0}(t)$ and $R\left(t, Q^{2}\right)$ starting from some initial value of rapidity $t_{0}$. In order to do this we place the initial dipole at the impact parameter $b \gg R_{0}\left(t_{0}\right)$. We then follow the evolution of $N(r, b)$. We show that:

a). Initially, as long as $b \geq R_{0}(t)$ the main contribution to the growth of $N(r, b)$ in eq.(1) comes from the points in the central region $|z-x|=|z-y| \simeq b$ with $z<R_{0}(t)$ on the right hand side of eq.(11). This long range contribution leads to exponential growth $R_{0}^{2}(t) \propto \exp \left\{\bar{\alpha}_{s} \epsilon t\right\}$. It also generates a power like tail in the scattering probability $N(r, b) \propto r^{2} / b^{2}$ and a nonvanishing saturation scale $Q_{s}(b)$ in the faraway region of impact parameters.

b). After $R_{0}(t)$ reaches the impact parameter $b$, further evolution in the vicinity of $b$ is dominated by local contributions from $|z-y| \simeq|z-x| \ll b$. These do not affect the growth of $R_{0}(t)$ but instead lead to a fast growth of $Q_{s}(b)$. As a result it takes only a short time for $Q_{s}(t, b)$ to reach the value $Q$, so that $R\left(t, Q^{2}\right)$ grows exponentially in $t$. 
We explain that the analysis of Ref. [⿴囗⿰丿㇄口⿺ fails on the following point. The initial dipole in Ref. $\left[\begin{array}{ll}\text { in in } \\ \text { in }\end{array}\right.$ the "grey" region at $R\left(t_{0}, Q^{2}\right)<b<R_{0}\left(t_{0}\right)$. This set up by construction disregards the evolution in the regions of transverse plane where the growth is dominated by the long range contributions. It therefore $a b$ initio misses the most important contributions to the growth of the total cross section. The crucial issue is not how fast the "grey" region becomes "black", but instead how fast the "white" region becomes "grey". By placing the initial dipole in the "grey" region, the authors of Ref. 河 prevent themselves from addressing this question.

We start by discussing some general properties of the scattering probability $N(x, y)$.

\section{Properties of $N(x, y)$}

The dipole scattering probability $N(x, y)$ depends on the size $r=x-y$ of the dipole $\{x, y\}$ and on its impact parameter $b=\frac{1}{2}(x+y)$ with respect to the hadronic target. For sufficiently small $b$ and $r$, it may be characterized in terms of the saturation scale $Q_{s}(t, b)$

$$
\begin{aligned}
N(x, y) & =\frac{1}{N_{c}} \operatorname{Tr}\left\langle 1-U^{\dagger}(x) U(y)\right\rangle \\
& \simeq 1-\exp \left[-Q_{s}^{2}(t, b)(x-y)^{2}\right] .
\end{aligned}
$$

It is important to keep in mind that the last equality only holds for small impact parameters. This last equality implies that for sufficiently large dipole size $r$, the scattering probability always approaches unity. However in reality at large enough impact parameters the scattering probability is perturbatively small for dipoles of arbitrary size. This follows since the colour fields far from the hadron vanish and thus $U(x) \simeq 1+O(g)$. Hence $N(x, y) \simeq O\left(\alpha_{s}\right) \ll 1$. Thus, for sufficiently large impact parameter, there is no transverse scale $1 / Q_{s}(t, b)$ above which the dipole scattering probability is of order unity, in contrast to the last line in (3).

To clarify the limited applicability of the concept of saturation scale $Q_{s}(t, b)$ in characterizing the dipole scattering probability, we illustrate the $r$ - and $b$-dependence of $N(x, y)$ in more detail:

Consider first a small dipole $r \ll R_{0}(t)$ where $R_{0}(t)$ denotes the radius within which the target colour fields are large at rapidity $t$. In this case, the two legs $x$ and $y$ of the dipole see the target field of approximately the same normalized strength $S(b)$, and the scattering probability can thus be written in the factorized form

$$
N(x, y)=n(r / L(b)) S(b) .
$$

Here, $L(b)$ characterizes the scale of correlations of the target field (correlation length) as a function of impact parameter. For impact parameter $b<R_{0}(t)$ where target colour fields are large, the colour field correlation length is equivalent to the inverse saturation scale, $L(b)=1 / Q_{s}(t, b)$. For larger impact parameters the correlation scale $L(b)$ is still a meaningful concept but $Q_{s}(t, b)$ does not exist.

At initial rapidity $t_{0}$, a physically sensible choice of the target field strength at impact parameter $b$ is

$$
\begin{aligned}
& S(b)=1 \quad \text { for } \quad b<R_{0}\left(t_{0}\right) \\
& S(b) \simeq \exp \left[-M\left(b-R_{0}\left(t_{0}\right)\right)\right] \quad \text { for } \quad b>R_{0}\left(t_{0}\right)
\end{aligned}
$$

where $M$ is some mass scale. One should keep in mind, however, that even though this initial profile shows an exponential fall-off at large distances, there is no guarantee that this functional shape is preserved in the evolution via (1). In fact, as seen in eq. (9) below, it is not.

As for the properties of the function $n$ in (4), one expects that it is a monotonically increasing function of its argument. Since $N(x, y)$ is always smaller than unity and since its maximal value is set by the strength of the target colour field, we have $n(r / L(b)) \leq 1$. For small dipoles, the scattering probability is vanishingly small and grows as $r^{2}$. Thus even the central region of the target is transparent for sufficiently small dipoles. For larger dipoles of size $r \propto L(b)$ the central region is black. However, for the profile (5.6) the last line of (3) holds only as long as $S(b)=1$, or $b \leq R_{0}$. In short, the initial scattering probability $N\left(t_{0}, x, y\right)$ can be parameterized in terms of some saturation scale $Q_{s}\left(t_{0}, b\right)$ only for impact parameters lying in the "grey" region of the target, at $b<R_{0}\left(t_{0}\right)$. The same of course applies also to scattering probability at any given value of $t$.

The situation is very different for dipoles of large size where the factorization (4) does not hold. This is the case e.g. for dipoles with $r=b$ sitting at large impact parameter $b \gg R_{0}(t)$. If $\vec{r}$ and $\vec{b}$ are orthogonal, then both legs of the dipole are in the "white" region (i.e., $\left.x, y \gg R_{0}(t)\right)$ and the scattering probability is small, $N(x, y) \ll 1$. If $\vec{r}$ and $\vec{b}$ are parallel instead, then one of the legs is in the "grey" region (i.e., $x<R_{0}(t)$ ) while the other is in the "white" region (i.e., $y \gg R_{0}(t), U(y) \simeq$ $1)$. As a consequence, the dipole scattering probability loses in this latter case any dependence on the size of the dipole and its impact parameter. It can be viewed as the scattering probability $1-\frac{1}{N_{c}} \operatorname{Tr}\left\langle U^{\dagger}(x)\right\rangle$ of a coloured probe in the sense

$$
\begin{aligned}
N(x, y) & =\frac{1}{N_{c}} \operatorname{Tr}\left\langle 1-U^{\dagger}(x) U(y)\right\rangle \\
& \simeq 1-\frac{1}{N_{c}} \operatorname{Tr}\left\langle U^{\dagger}(x)\right\rangle, \quad \text { for } \quad y \gg R_{0}(t) .
\end{aligned}
$$

For $x<R_{0}(t)$ the target fields are large, and $\langle U(x)>=$ 0 . Thus $N(x, y)=1$. Accordingly, the radius $R_{0}(t)$ characterizes the size of the black disk for a coloured probe. Again, for dipoles for which (7) applies, the scattering 
probability $N(x, y)$ cannot be characterized in terms of a saturation scale $Q_{s}$.

To sum up, the saturation scale $Q_{s}(t, b)$ is a meaningful concept only in the region of impact parameter space which is black for a coloured probe, $b<R_{0}(t)$. For larger impact parameter, the scattering probability is smaller than unity for arbitrarily large dipoles and the last line of (3) does not apply.

\section{Long-range Contributions violate the Froissart Bound}

We consider now a small-size dipole $r=x-y=1 / Q \ll$ $R_{0}\left(t_{0}\right)$ which has at initial rapidity $t_{0}$ a negligible interaction probability with the hadronic target since it sits at large impact parameter $b \gg R_{0}\left(t_{0}\right)$. We ask how rapidly its scattering probability grows to order unity as a function of rapidity $t$. We first establish that the initial growth of $N(x, y)$ is dominated by long-range contributions to the $z$-integral in (11).

If the newly generated dipole leg $z$ in eq.(1) sits at transverse position in the neighbourhood of $x, y$, i.e. in the white region, then $N(x, z) \sim N(y, z) \sim N(x, y) \ll 1$ and the non-linear term in the BK equation can be neglected. As a consequence, the short-range contribution grows proportional to the local scattering probability which was taken to have an exponential fall-off at initial rapidity,

$$
\left.\frac{d}{d t} N(x, y)\right|_{\text {short }} ^{\text {at } t=t_{0}} \propto \alpha_{s} e^{-M\left(b-R_{0}\left(t_{0}\right)\right)} .
$$

For the long-range contribution, a lower bound of the growth of $N(x, y)$ can be obtained by considering contributions from the "grey" region only. With the initial dipole in the white region (i.e. $N(x, y) \simeq 0$ ) but the leg $z$ in the "grey" region (i.e. $N(x, z) \simeq 1, N(y, z) \simeq 1$ ), the BK equation (11) gives

$$
\left.\frac{d}{d t} N(x, y)\right|_{\text {long }}=\bar{\alpha}_{s} \frac{r^{2}}{b^{4}} R_{0}^{2}(t) .
$$

Comparison of eqs. (8) and (9) shows that at sufficiently large impact parameter $b \gg R_{0}(t)$, the dominant growth of the dipole scattering probability comes from long-range contributions.

It is important that $R_{0}(t)$ appearing on the right hand side of (9) is the radius of the black region for a coloured probe in the precise sense specified in (7). Namely, while the right hand side of (11) depends on colourless dipoles $\{x, y\},\{x, z\}$ and $\{y, z\}$, only one of their coloured legs $z$ touches the target. Thus, the scattering probability for these dipoles depends only on the strength of the target-induced colour rotation $1-\frac{1}{N_{c}} \operatorname{Tr}\left\langle U^{\dagger}(z)\right\rangle$ for this one coloured leg, i.e. it is the scattering probability of a coloured probe.

As shown in Ref. 1, 2] and as rederived in Ref. [4, the black disk radius $R_{0}(t)$ for a coloured probe grows exponentially with rapidity,

$$
R_{0}(t)=R_{0}\left(t_{0}\right) \exp \left[\bar{\alpha}_{s} \epsilon\left(t-t_{0}\right)\right]
$$

Substituting this into eq.(9) and integrating up to rapidity

$$
t_{1}=\frac{1}{\bar{\alpha} \epsilon} \ln \frac{b}{R_{0}\left(t_{0}\right)}
$$

we find

$$
\left.N(r, b)\right|_{t_{1}} \sim \frac{r^{2}}{b^{2}}
$$

Thus, as advertized we find that by the time the radius of the "grey" region $R_{0}(t)$ reaches the impact parameter $b \gg R_{0}\left(t_{0}\right)$, the scattering probability $N(r, b)$ is not exponentially suppressed as suggested by eqs. (4, 6) but rather has a long power tail.

Since we expect $R\left(t, Q^{2}\right)<R_{0}(t)$, we can not use the approximation eq.(9) beyond the rapidity $t_{1}$. We can nevertheless establish the exponential growth of $R\left(t, Q^{2}\right)$. Starting at rapidity $t_{1}$, the point $b$ enters the "grey" region. From this point on the evolution is dominated by "local" contributions. The concept of saturation scale is well defined at $b$ for $t>t_{1}$. As shown in many previous works [5], and rederived in [4] the saturation momentum grows exponentially with rapidity

$$
Q_{s}(t, b)=Q_{s}\left(t_{1}, b\right) \exp \left[\bar{\alpha}_{s} \lambda\left(t-t_{1}\right)\right]
$$

Thus at rapidity $t_{2}$ such that

$$
t_{2}-t_{1}=\frac{1}{\bar{\alpha} \lambda} \ln \frac{Q}{Q_{s}\left(t_{1}, b\right)},
$$

the scattering probability $N(r, b)$ reaches unity.

Putting eqs.(11) and (14) together we find that the radius of the black disk $R\left(t, Q^{2}\right)$ reaches a given impact parameter $b$ at rapidity $t$

$$
t=\frac{1}{\bar{\alpha} \epsilon} \ln \frac{R\left(t, Q^{2}\right)}{R_{0}\left(t_{0}\right)}+\frac{1}{\bar{\alpha} \lambda} \ln \left[\frac{Q}{Q_{s}\left(t_{1}, b\right)}\right] .
$$

We have not calculated the exact value of $Q_{s}\left(t_{1}, b\right)$, but even without doing so we can put an upper bound on $t$. Whatever the value of $Q_{s}\left(t_{1}, b\right)$, it is greater than the inverse of the impact parameter $b^{-1}[\overline{6}]$. Thus substituting $b$ for $Q^{-1}\left(t_{1}, b\right)$ in eq.114) and adding eq.(11) we find the upper bound on the time that it takes the black disk $R\left(t, Q^{2}\right)$ to reach a given impact parameter $b$

$$
t<\frac{1}{\bar{\alpha} \epsilon} \ln \frac{R\left(t, Q^{2}\right)}{R_{0}\left(t_{0}\right)}+\frac{1}{\bar{\alpha} \lambda} \ln \left[R\left(t, Q^{2}\right) Q\right]
$$


Thus

$$
\sigma\left(t, Q^{2}\right) \sim 2 \pi R\left(t, Q^{2}\right)>2 \pi\left[R_{0}^{\lambda}\left(t_{0}\right) Q^{-\epsilon}\right]^{\frac{1}{\epsilon+\lambda}} e^{\frac{\epsilon \lambda}{\epsilon+\lambda} \bar{\alpha}_{s} t} .
$$

We stress that eq.(17) is the lower bound on $R\left(t, Q^{2}\right)$. For example in the case when $Q_{s}\left(t_{1}\right)$ is a fixed $b$-independent scale, the rates of growth of $R\left(t, Q^{2}\right)$ and $R_{0}(t)$ are the same.

\section{Critique of FIIM}

The mistake in the argument of Ref. [1] can now be easily understood. The set-up of Ref. [4] is in principle inadequate for studying the growth of $R\left(t, Q^{2}\right)$. In Ref. [4] the saturation scale at impact parameter $b$ is assumed to be defined and to be perturbatively large at initial rapidity $t_{0}, Q_{s}\left(t_{0}, b\right) \gg \Lambda_{\mathrm{QCD}}$. Thus, by definition, this set-up ignores evolution in the region $b>R_{0}\left(t_{0}\right)$ where the target field strength is initially small and where the saturation scale does not exist at initial rapidity $t_{0}$. As is clear from the discussion of eq. (9) above, the power tails generated in this region quickly start dominating the total cross section, and therefore cannot be ignored. It is this powerlike fall off of $N(b)$ (or equivalently $S(b)$ ) and not the initial exponential one, that has to be taken as the "basic distribution" which is further evolved by local effects. This has been ignored in Ref. [4].

The physics is quite simple. We know from Refs. 11. 2, 4h that $R_{0}(t)$ grows exponentially with $t$. Then eq.(12) tells us that for $R_{0}\left(t_{0}\right)<b<R_{0}(t)$, that is up to distances exponentially larger than the initial radius $R_{0}$, the long range contributions generate a powerlike tail for the scattering probability. This is to be contrasted with the exponential fall off assumed for the initial profile. We can now use the same argument as in Ref. 栣 to establish that the total cross section of a dipole of size $r=1 / Q$ grows exponentially. Starting from rapidity $t_{1}$, where $t_{1}$ is given in eq.(11), the evolution of $N(r, b)$ at the impact parameter $b$ is governed by the BFKL equation. The initial condition for this BFKL evolution at $t_{1}$ is furnished by the previous evolution from $t_{0}$ to $t_{1}$ which is dominated by the long range contributions to eq.(11). This initial condition is given by eq.(12). Thus all we have to do is to replace in eqs.(4.5) of Ref. 吽 the exponential tail $\exp \left[-2 m_{\pi} b\right]$ by the power law tail $r^{2} / b^{2}=\left(Q^{2} b^{2}\right)^{-1}$ (see eq.(12)) and take into account the fact that the BFKL evolution goes on not for the whole rapidity interval $t$, but rather only for $t-t_{1}$. Neglecting the so called diffusion term as in Ref.[4] we obtain:

$$
N\left(Q^{2}, b\right)=\exp \left[-\ln \left[Q^{2} b^{2}\right]+\omega \bar{\alpha}_{s}\left(t-t_{1}\right)-\frac{1}{2} \ln \frac{Q^{2}}{\Lambda^{2}}\right] .
$$

Equating the scattering probability to one at $b=$
$R\left(t, Q^{2}\right)$, we obtain

$$
R\left(t, Q^{2}\right)=\left[\left[\frac{\Lambda}{Q^{3}}{ }^{\epsilon} R_{0}\left(t_{0}\right)^{\omega}\right]^{\frac{1}{\omega+2 \epsilon}} e^{\frac{\omega \epsilon}{\omega+2 \epsilon} \bar{\alpha}_{s} t} .\right.
$$

Here $\Lambda$ is the saturation momentum in the central region of the target at the initial value of rapidity. Given that $2 \lambda<\omega$, eq. (19) is consistent with the lower bound eq.(17). Thus we see again that the radius of the black disk for the scattering of a colourless dipole grows exponentially with $t$. The result of Refs. [1, 2] stands confirmed.

\section{Final comments}

We finally give an intuitive physics argument of why the total cross section of a colourless dipole violates the Froissart bound if it is violated by a coloured scattering probe. To this end, we first recall an argument due to Heisenberg: consider a theory with a mass gap, where the profile of the distribution of matter density in any target must decay exponentially at the periphery, $\rho(b) \propto \exp [-m b]$. As this target is struck by a projectile, in order to produce an inelastic scattering event at least one particle must be produced. Assuming that the scattering is local in the impact parameter plane, the region of the overlap of the probe and the target must therefore contain energy at least equal to the mass of the lightest particle, $m$. For scattering at energy $E=s / m$ in the frame where all the energy resides in the target, the target energy density is $E \rho(b)$. Thus the scattering can only take place for impact parameters smaller than those that satisfy $E \exp [-m b]=m$. Thus $b_{\max }=\frac{1}{m} \ln \left[\mathrm{s} / \mathrm{m}^{2}\right]$, which is equivalent to the Froissart bound. Conversely, if the cross section grows as a power of energy, then the density distribution in the target is not exponential but power like. With $\rho(b) \propto b^{-\lambda}$ one obtains $b_{\max } \propto s^{\frac{1}{\lambda}}$ and this violates the Froissart bound. Assume now that the total cross section for a coloured probe (a quark, say) violates unitarity, i.e., the scattering probability for a quark at impact parameter $b$ is proportional to $\rho(b) \propto \frac{1}{b^{\lambda}}$. A quark-antiquark dipole of transverse size $r$ will compare the target field strength at two points separated by the distance $r$. Its scattering probability is thus proportional to $r \frac{d}{d b} \rho(b) \propto r \frac{1}{b^{\lambda-1}}$, which is still a power-law fall-off. Hence, the total dipole-hadron cross section violates the Froissart bound if the total quark-hadron cross section does so.

\section{Note added}

After the appearance of the present paper, the authors of [4] added a note to [4] agreeing with our conclusions. Unfortunately they also attribute to the present paper as 
well as to our previous papers [1], [2] several statements which we never made nor intended to make. For example, they attribute to our works "the statement that one cannot compute the high-energy cross section using the ideas of (perturbative) saturation". To the contrary, it is the main point of Ref. [2] to explain how the ideas of perturbative saturation can be used for the calculation of high-energy cross sections. For lack of space, we refrain here from giving further examples of the same kind. Although Ref. [4] is quite misleading in the way it presents some aspects of our work, we are confident that the careful reader will easily identify these inaccuracies.

[1] A. Kovner and U. A. Wiedemann, Phys. Rev. D 66 (2002) 051502 arXiv:hep-ph/0112140].
[2] A. Kovner and U. A. Wiedemann, Phys. Rev. D 66 (2002) 034031 arXiv:hep-ph/0204277.

[3] I. Balitsky Nucl. Phys. B463 (1996) 99; Yu. Kovchegov Phys. Rev. D60 (1999) 034008.

[4] E. Ferreiro, E. Iancu, K. Itakura and L. McLerran, arXiv:hep-ph/0206241.

[5] See for example E. Levin and K. Tuchin Nucl. Phys. B573 (2000) 833; Nucl. Phys. A693 (2001) 787.

[6] The value $Q_{s}^{-1}(t, b) \sim b$ is suggested by eq. (12). However since eq.(12) is the lower bound on $N(r, b)$, the value $b$ is also a lower bound on $Q^{-1}(t, b)$. 